\title{
Capacity and Delay Analysis in Delay Tolerant Network with Multiple Communities
}

\author{
Yahui Wu, Su Deng, Hongbin Huang
}

\begin{abstract}
This paper studies the capacity and delay of delay tolerant network (DTN) with multiple communities. Due to the impact of social selfishness, nodes in DTN are more willing to forward messages coming from the same community. In such a scenario, this paper first mathematically defines the maximal capacity, and then proposes a single copy two-hop routing algorithm which can achieve the maximal capacity. Furthermore, it proposes a theoretical model to evaluate the delay of this algorithm. To decrease the delay, this paper modifies above algorithm, and assumes that nodes just forward messages to nodes in the same community. The capacity and delay of this new algorithm are also obtained. Numerical results show that the selfish behavior decreases the capacity and increases the delay rapidly. However, the new algorithm can decrease the delay at the cost of decreasing the capacity. This result shows that if the sources just
\end{abstract} forward their messages to nodes in the same community, the delay may decrease.

Index Terms - Delay tolerant network, Capacity and delay, Multiple Communities, Social Selfishness

\section{INTRODUCTION}

$\mathrm{C}$ APACITY and delay analysis is popular since the famous work by Gupa and Kumar [1], which gets the network capacity of a static network. Then, Grossglauser and Tse study the capacity and delay in a mobile wireless network, and they find that the mobility can increase the capacity [2]. However, in work [2], the delay may become infinite, so it is not proper in the real applications. For this reason, the capacity and delay trade-off is explored in [3]. The work [4] studies the capacity and delay in a network with correlated mobility.

The delay tolerant network (DTN) is often used in the scenarios, where the communication paths may not exist at given time [5]. To overcome this problem, nodes in DTN adopt the store-carry-forward communication mode. In this mode, nodes will first store the message in their buffer, and carry along their movement. When a new communication opportunity occurs, the message will be forwarded to other nodes. It is easy to see that such communication mode closely depends on the cooperation between nodes.

Manuscript received July, 2014.

Yahui Wu, Su Deng, and Hongbin Huang are with the Science and Technology on Information Systems Engineering Laboratory, National University of Defense Technology, Changsha 410073 China. Email: wuyahui@nudt.edu.cn. Research reported in this paper is supported by NSF No. 61401482. 
In the real applications, nodes are often divided into multiple communities according to certain social ties [6]. On the other hand, nodes may be selfish, and may be more willing to help the one in the same communities. This behavior is denoted as the social selfishness [7]. At present, there are many works which get different theoretical models to evaluate the impact of the social selfishness in different scenarios [7]-[8]. However, these works often assume that there is just one source and the source just creates one single message. Therefore, these models cannot be used to evaluate the impact of social selfishness on the capacity and delay.

Based on above description, this paper gets some new contributions, which are summarized as follows.

1. This paper obtains the accurate model about the maximal capacity of DTN with multiple communities. In particular, it considers the social selfishness.

2. This paper proposes a single copy two-hop routing algorithm (Algorithm 1), and shows that this algorithm can achieve the maximal capacity. Then, the theoretical model about the delay is obtained.

3. This paper obtains algorithm2 by modifying algorithm1. In this algorithm, nodes just forward messages towards nodes in the same community. The capacity and delay of this algorithm are also obtained.

4. Numerical results show that the social selfishness will decrease the capacity and increase the delay. However, Algorithm 2 can decrease the delay at the cost of smaller capacity. This means that if the users want to improve the delay, they can make the packets transmitted just within their own community.

\section{NETWORK MODEL}

\section{A. Mobility Model}

In this paper, we assume that there are totally $n$ nodes, which are divided into $k$ non-overlapping communities. These communities are denoted as $1,2, \ldots k$. Let $n_{i}$ denote the number of nodes in $i$-th community, and then we have,

$$
\sum_{i=1}^{k} n_{i}=n
$$

In DTN, the messages can be transmitted between two nodes only when they meet, so the mobility patterns are critical. At present, there are many works which explore the theoretical models to fit the mobility patterns. However, the mobility patterns are very sophisticated, so the common model to fit all the motion traces does not exists. For this reason, various models are proposed, which can be used in different scenarios, such as the Home-MEG model [9], Edge-Markov model [10], etc. Though these models can fit some real motion traces very well, they are too complex. Therefore, we use the exponential model which is very simple [7]-[8]. In this model, the inter-meeting time between two consecutive contacts follows the exponential distribution with parameter $\beta$. Thus, two nodes meet each other in time interval $\Delta t$ with probability $1-\exp (-\beta \Delta t)$. Such model has been used in many works [7]-[8]. In addition, many works show that this model is accurate in some applications. For example, [11]-[12] describe the inter-meeting time of human or vehicles by exponential distribution and validate their model experimentally on real motion traces. 


\section{B. Social selfishness}

Due to the social selfishness, we can assume that nodes in $i$-th community forward messages to nodes in $j$-th community with probability $p_{i j}\left(0 \leq p_{i j} \leq 1\right)[7]$-[8]. In addition, nodes in community $i$ receive messages coming from nodes belonging to $j$-th community with probability $p_{i j}$, too.

Other assumptions: The source and its destination do not show any selfish behavior for packets of their own flow. On the other hand, we have $p_{i j}=p_{j i}$. Our work can be extended to the case without these assumptions easily.

\section{Traffic Pattern}

Similar to [3], we adopt the permutation traffic pattern. In this pattern, there are $n$ source-destination pairs and the destinations in any different pairs are different. For simplicity, we assume that the source and its destination are in the same community. Our work can be extended to other cases easily.

Each source-destination pair has an information flow, and the packet arrival process at the source belonging to $i$-th community is a Poisson process with rate $\lambda_{i}>0$ [3]. For each source-destination pair, other nodes can be seen as relay nodes.

\section{Some Definitions}

Definition of capacity: For a fixed packet arrival rate $\lambda_{i}$, the network is stable if a scheduling algorithm exists and in this algorithm the queue in each node does not increase to infinity as time goes to infinity. Then, the per-node capacity is the maximal arrival rate that the network can remain stable [3]. The network capacity is the sum of all nodes' per-node capacity.

In this paper, we use capacity to denote the network capacity simply. The following is the definition of delay.

Definition of delay: The delay of a packet equals to the time it reaches to the destination minus the time it arrives at the source.

\section{CAPACITY AND DELAY ANALYSIS}

In this section, we first present the maximal capacity and prove the necessity. Then, the sufficiency is proved by proposing a single-copy two-hop routing protocol. In addition, the delay of this algorithm is obtained, too. Finally, we propose Algorithm 2 and study its capacity and delay.

Theorem 1. For the network described above, the maximal capacity $\mu$ is denoted as

$$
\mu=\frac{\beta n}{2}+\sum_{i=1}^{k} n_{i}\left(\frac{\beta\left(n_{i}-2\right)}{4} p_{i i}+\sum_{j=1, j \neq i}^{k} \frac{\beta n_{j}}{4} p_{i j}\right)
$$

Similar to [3], the proof consists of proving that $\lambda \leq \mu$ is necessary and $\lambda<\mu$ is sufficient.

\section{A. Proof of Necessity}

Let $X_{h}(T)$ denote the number of packets that reach to the destinations through $h$ hops in time interval $[0, T]$. Let $Y(T)$ denote the 
number of all transmissions in time interval $[0, T]$.

It is easy to see that if the network is stable, the overall output rate should be not lower than the overall input rate [3]. The input rate equals to the overall arrival rate of the packets of all the flows. In addition, the output rate equals to the overall rate of the packets reaching to the destinations. Then, we can get

$$
\frac{\sum_{h=1}^{\infty} X_{h}(T)}{T} \geq \sum_{i=1}^{k} n_{i} \lambda_{i}-\varepsilon, \varepsilon>0 .
$$

Furthermore, we have,

$$
Y(T) \geq \sum_{h=1}^{\infty} h X_{h}(T) \geq X_{1}(T)+2 \sum_{h=2}^{\infty} X_{h}(T) .
$$

Combining with Eq.(3), Eq.(5) follows.

$$
\sum_{i=1}^{k} n_{i} \lambda_{i} \leq \frac{X_{1}(T)}{2 T}+\frac{Y(T)}{2 T}+\varepsilon
$$

$X_{1}(T)$ denotes the number of packets that reach to the destinations through 1 hop in time interval $[0, T]$. Therefore, $X_{1}(T) / T$ denotes the rate of the packets reaching to destinations through 1 hop in time interval $[0, T]$. We define the contact rate as the number of contacts between nodes in a unit time. There are $n$ nodes and any two nodes encounter with rate $\beta$, so the total contact rate is $\beta n(n-1) / 2$. For any two nodes $i$ and $j$, they are source-destination pair with probability $1 / n-1$. Therefore, the contact rate between the source-destination pairs is $\beta n / 2$. Each time the source meets a destination, a packet will be forwarded to the destination. Therefore, we can obtain,

$$
\frac{X_{1}(T)}{T} \rightarrow \frac{\beta n}{2}
$$

Then, we can easily find that the number of transmissions between these pairs is $T \beta n / 2$.

Besides the transmissions between the source-destination pairs, other transmissions at least include one relay node. For nodes in community $i$, the number of such contacts is $T \beta n_{i}\left(n_{i}-1\right) / 2-T \beta n_{i} / 2=T \beta n_{i}\left(n_{i}-2\right) / 2$. For such contact, there is at least one relay node, so at least one node shows the selfish behavior. Nodes in the same community forward or receive messages with probability $p_{i i}$, so the number of such transmissions is at most $T \beta n_{i}\left(n_{i}-2\right) p_{i i} / 2$.

Similarly, the number of contacts between nodes in communities $i$ and $j$ is $\beta n_{i} n_{j} / 2$. Because we have assumed that the source and its destination are in the same community, these contacts include the relay nodes. Thus, they will show the selfish behavior. Because any of these contacts is formed by nodes in community $i$ and $j$, the selfish probability is $p_{i j}$. Then, the total number of transmissions is at most $T \beta p_{i j} n_{i} n_{j} / 2$.

Based on above analysis, we can easily obtain

$$
\frac{Y(T)}{T} \leq \frac{\beta n}{2}+\frac{\sum_{i=1}^{k} \beta n_{i}\left(\left(n_{i}-2\right) p_{i i}+\sum_{j=1, j \neq i}^{k} n_{j} p_{i j}\right)}{2}
$$


Combining with Eq.(5), Eq.(6) and Eq.(7), Eq.(8) follows.

$$
\sum_{i=1}^{k} n_{i} \lambda_{i} \leq \frac{\beta n}{2}+\sum_{i=1}^{k} n_{i}\left(\frac{\beta\left(n_{i}-2\right)}{4} p_{i i}+\sum_{j=1, j \neq i}^{k} \frac{\beta n_{j}}{4} p_{i j}\right)+\varepsilon
$$

Because $\varepsilon$ can be arbitrarily small, Eq.(2) follows.

\section{B. Proof of Sufficiency}

In this section, we propose the single copy two-hop routing algorithm (Algorithm 1), in which each node has two queues. The source queue stores the locally generated packets, and the relay queue stores the packets coming from other sources. For simplicity, in this paper, if a queue stores the packets generated by $S$, we can say that the queue is for $S$. When two nodes meet (e.g., $g$ and $f$, belonging to communities $i$ and $j$, respectively), each node is selected as the transmitter with probability $1 / 2$. Thus, the other node is the receiver. For simplicity, we can assume that $g$ is the transmitter and $f$ is the receiver. If $f$ is the destination of $g$, $g$ carries out the source-destination transmission. Otherwise, $g$ carries out the source-relay transmission and relay-destination transmission with equal probability (the probability is $1 / 2$ ).

1. source-destination: $g$ selects the head of line packet (e.g., $P_{h}$ ) from its source queue, and forwards it to $f$. Then, $g$ deletes the packet. After this process, the packet behind $P_{h}$ will be head of line.

2. source-relay: $g$ selects the head of line packet (e.g., $P_{h}$ ) from its source queue, and forwards it to $f$. However, $f$ just receives this packet with probability $p_{i j}$. If the transmission is successful, $g$ will delete $P_{h}$, and the packet behind $P_{h}$ will be head of line. After receiving $P_{h}, f$ will put $P_{h}$ into the end of its relay queue.

3. relay-destination: $g$ selects the head of line packet (e.g., $P_{h}$ ) from its relay queue, and forwards the packet to $f$ with probability $p_{i j}$. Because $f$ is the packet's destination, it receives the message immediately. If the transmission is successful, $g$ will delete $P_{h}$.

Lemma 1. Under Algorithm 1, the average delay of packets coming from the sources in community $i(1 \leq i \leq k)$ can be denoted as

$$
E\left(W_{i}\right)=\frac{(n-1)}{\mu_{i}-\lambda_{i}} .
$$

Proof: We assume that a source $S$ in community $i$ is serving for the packet $P_{h}$. According to Algorithm $1, P_{h}$ will leave from $S$ if $P_{h}$ is transmitted to other nodes.

Note that $S$ meets its destination with rate $\beta$, and $S$ is selected as the transmitter with probability $1 / 2$. Because there is no selfish behavior between the source and destination, $P_{h}$ will be transmitted to its destination with rate $\beta / 2$.

If $S$ meets a relay node (e.g., $R$ ), it carries the source-relay transmission with probability $1 / 4$.

It is easy to see that $S$ meets the relay nodes in the same community with rate $\beta\left(n_{i}-2\right)$. Because $P_{h}$ belongs to the flow of $S, S$ does not have the selfish behavior. However, the receiver is the relay node, so it receives the packet with probability $p_{i i}$. Therefore, $S$ forwards the packet to relay nodes in the same community with rate $\beta\left(n_{i}-2\right) p_{i i} / 4$. 
Similarly, $S$ meets nodes (e.g., $R$ ) in community $j$ with rate $\beta n_{j}$. Because $S$ and $R$ are in different communities, $R$ is the relay node and its selfish probability is $p_{i j}$. Therefore, the transmission rate is $\beta n_{j} p_{i j} / 4$.

Then, we can see that the total severing rate is $\beta / 2+\beta\left(n_{i}-2\right) p_{i i} / 4+\beta n_{j} p_{i j} / 4$, and it is also the output rate $\mu_{i}$ of the source queue of $S$. Therefore, if $\lambda_{i}<\mu_{i}$, the source queue is stable.

For the relay queue, it can be divided in to $n-1$ sub-relay queues, and each corresponds to one of the other $n$ - 1 sources. Here, we just consider the sub-relay queue $Q S$ for the source $S$. In other words, the queue $Q S$ stores the messages generated by $S$. In addition, all of the relay nodes have the queue $Q S$.

According to Burke's theorem [13], the output process of the source queue of $S$ is also a Poisson process with rate $\lambda_{i}$. Therefore, The packets leaving from $S$ reaches to the nodes in community $i$ with rate $\lambda_{i}\left(\beta\left(n_{i}-2\right) p_{i i} / 4\right) / \mu_{i}$. For a given packet, a specific relay node (e.g., $R$ ) in community $i$ gets the packet with probability $1 /\left(n_{i}-2\right)$. Then, the packets leaving from $S$ reach to the $Q S$ of $R$ with rate $\lambda_{i} \beta p_{i j} / 4 \mu_{i}$, which is also the input rate of $Q S$. Similarly, we can get the input rate of the $Q S$ of nodes in community $j$ which equals to $\lambda_{i} \beta p_{i j} / 4 \mu_{i}$

Because there is only one copy, at any time there is at most one node carrying the packet $P_{h}$. Suppose that $R$ gets the message. In this case, if $R$ is in community $i$, it forwards the packet to its destination with rate $\beta p_{i i} / 4$. Otherwise, the rate is $\beta p_{i j} / 4$. These are the output rates of $Q S$.

Therefore, if the sub-relay queue $Q S$ is stable, we must have $\lambda_{i} \beta p_{i j} / 4 \mu_{i}<\beta p_{i j} / 4,1 \leq j \leq k$. Then, we can find that if $\lambda_{i}<\mu_{i}$, $\lambda_{i} \beta p_{i j} / 4 \mu_{i}<\beta p_{i j} / 4$ follows. This means that if $\lambda_{i}<\mu_{i}$, both the source queue and the sub-relay queue for sources in community $i$ are stable. We can obtain similar result for sources in other communities. On the other hand, the sub-relay queues for different sources are independent. Therefore, if $\lambda_{i}<\mu_{i}(1 \leq i \leq k)$, the network is stable.

Based on above analysis, we can see that if the packet is obtained by the destination directly, the average delay $E\left(W_{i}\right)$ equals to the residence time at the source queue. Otherwise, $E\left(W_{i}\right)$ equals to the sum of the residence time at the source queue and the residence time at the sub-relay queue, so we have

$$
E\left(W_{i}\right)=E\left(T_{S}\right)+\frac{\frac{\beta\left(n_{i}-2\right)}{4} p_{i i}}{\mu_{i}} E\left(T_{i}\right)+\frac{\sum_{j=1, j \neq i}^{k} \frac{\beta n_{j}}{4} p_{i j} E\left(T_{j}\right)}{\mu_{i}} .
$$

$E\left(T_{S}\right)$ and $E\left(T_{j}\right)$ denote the residence times at the source queue and the sub-relay queue of the relay node in community $j(1 \leq j \leq k)$, respectively.

According to little's theorem, we have $E\left(T_{S}\right)=1 /\left(\mu_{i}-\lambda_{i}\right)$. Similarly, we can obtain $E\left(T_{j}\right)=1 /\left(\beta p_{i j} / 4-\lambda \beta p_{i j} / 4 \mu_{i}\right), 1 \leq j \leq k$. Thus, combining with Eq.(10), Eq.(9) follows.

It is easy to see that the sum of all nodes' output rate equals to $\mu$ in Eq.(2). Based on Lemma 1, the Sufficiency follows. 


\section{Transmission within the Same Community}

Now, we propose Algorithm 2, in which the source just forwards its messages to nodes in the same community. Other steps are the same as that in Algorithm 1.

Theorem 2. The capacity and delay of Algorithm 2 are

$$
\begin{gathered}
\mu_{\text {new }}=\sum_{i=1}^{k} n_{i} \mu_{\text {new }, i} \leq \sum_{i=1}^{k} n_{i}\left(\frac{\beta}{2}+\frac{\beta\left(n_{i}-2\right)}{4} p_{i i}\right) \\
=\frac{\beta n}{2}+\sum_{i=1}^{k} n_{i}\left(\frac{\beta\left(n_{i}-2\right)}{4} p_{i i}\right) . \\
E\left(W_{\text {new }, i}\right)=\frac{1}{\mu_{\text {new }, i}-\lambda_{i}}+\frac{\left(n_{i}-2\right)}{\mu_{\text {new }, i}-\lambda_{i}}=\frac{\left(n_{i}-1\right)}{\mu_{\text {new }, i}-\lambda_{i}}, i \in[1, k] .
\end{gathered}
$$

$\mu_{\text {new }}$ and $\mu_{\text {new }, i}$ denote the capacity and capacity of nodes in community $i$ of the new algorithm, respectively. $E\left(W_{\text {new, } i}\right)$ denotes the average delay of packets coming from nodes in community $i$.

The proof of Theorem 2 is the same as Theorem 1, and we do not present the details here.

\section{NUMERICAL RESULTS}

First, we consider the capacity in different cases. Here, we assume that there are $n$ nodes, and they are evenly divided into 4 communities $\left(k=4, n_{i}=n / 4,1 \leq i \leq k\right)$. Then, we set $\beta=3.71 \times 10^{-6} \mathrm{~s}^{-1}$, and this value comes from the Shanghai city motion trace, which is used in many works [14]. For the social selfishness, we assume that $p_{i i}=0.8, p_{i j}=0.2,1 \leq i, j \leq k, i \neq j$.

Through letting $n$ increase from 20 to 200, we get Fig.1. From the result, we can see that the capacity in Algorithm 2 is smaller. This means that if the packets are just transmitted within the community where they come from, the capacity will decrease.

Now, we begin to explore the average delay. Here, we let the total input rate $\lambda$ equal to 0.00009 . Other settings remain unchanged. Because the four communities have the same number of nodes and the same degree of social selfishness, we can easily find that the nodes have the same per-node capacity and the packets have the same average delay. For simplicity, for any node, we set its input rate as $\lambda / n$. In this case, the average delay is shown in Fig. 2.

The result in Fig. 2 shows that the average delay in Algorithm 2 is smaller. This means that if the packets are just transmitted within the community where they come from, the delay will decrease at the cost of decreasing the capacity.

Then, we set $n=200, p_{i i}=0.8 p, p_{i j}=0.2 p, 1 \leq i, j \leq k, i \neq j$. Other settings remain unchanged, let $p$ increase from 0.1 to 1 , and we get Fig.3 and Fig.4. From these figures we can find similar result as Fig.1 and Fig.2. In addition, Fig.3 and Fig.4 also shows the impact of the social selfishness. Note that $p_{i i}=0.8 p, p_{i j}=0.2 p, 1 \leq i, j \leq k, i \neq j$, so if $p$ is bigger, the cooperative level between nodes is bigger. Correspondingly, the social selfish level is smaller. As shown in Fig.3 and Fig.4, the capacity increases with $p$, so if the social selfish level is smaller, the network can get bigger capacity. Similarly, if the social selfish level is smaller, the average delay is smaller. Therefore, the social selfishness has significant impact. 


\section{CONCLUSION}

This paper first defines the maximal capacity. Then, a single copy two-hop routing algorithm (Algorithm 1) is proposed, which can achieve the maximal capacity. The accurate model about the average delay of Algorithm 1 is given, too. Based on Algorithm 1, Algorithm 2 is proposed, in which the sources just forward their packets to nodes in the same community. The capacity and average delay of Algorithm 2 are also presented. Numerical results show that the social selfishness will decrease the performance. However, Algorithm 2 can improve the delay at the cost of decreasing the capacity. This means that if smaller delay is more important, the sources should not forward their packets to nodes in other communities.

\section{REFERENCES}

[1] P. Gupta, and P. R. Kumar, "The capacity of wireless networks," IEEE Transactions on Information Theory, vol.46, no.2, pp.388-404, 2000.

[2] M. Grossglauser, and D. N. C. Tse, "Mobility increases the capacity of ad hoc wireless networks," ACM/IEEE Transactions on Networking, vol.10, no.4, pp.477-486, 2002.

[3] M. J. Neely, and E. Modiano, "Capacity and delay tradeoffs for ad hoc mobile networks," IEEE Transactions on Information Theory, vol.51, no.6, pp.1917-1937, 2005.

[4] S. Yao, X. Wang, X. Tian, and Q. Zhang, "Delay-Throughput Tradeoff with Correlated Mobility of Ad-Hoc Networks," in Proc. of INFOCOM, 2014.

[5] K. Fall, "A delay-tolerant network architecture for challenged internets," in Proc. of ACM SIGCOMM, pp.27-34, 2003.

[6] N. P. Nguyen, T. N. Dinh, Y. Xuan, and M. T. Thai, "Adaptive algorithms for detecting community structure in dynamic social networks," in Proc. of INFOCOM, pp. 2282 - 2290, 2011.

[7] Y. Li, P.Hui, D. Jin, L. Su, L. Zeng, "Evaluating the impact of social sefishness on the epidemic routing in delay tolerant networks," IEEE Communication Letters, 14(11):1026-1028, 2010.

[8] Y. Li, G. Su, D. Wu, D. Jin, L. Su, and L. Zeng, "The impact of node selfishness on multicasting in delay tolerant networks," IEEE Transactions on Vehicular Technology, 60(5):2224-2238, 2011.

[9] A. Clementi, R. Silvestri and L. Trevisan, "Information spreading in dynamic graphs," in Proc. ACM PODC, pp.27-46, 2012.

[10] A. Clementi, C. Macci, A. Monti, F. Pasquale and R. Silvestri, "Flooding time of edge-markovian evolving graphs," SIAM Journal on Discrete Mathematics, vol.24, no.4, pp.1694-1712, 2010.

[11] T. Karagiannis, L. Boudec, and M. Zojnovic, "Power law and exponential decay of inter contact times between mobile devices," IEEE Transactions on Mobile Computing, vol. 9, no.10, pp. 1377-1390, 2010.

[12] H. Cai, D. Eun, "Crossing over the bounded domain: from exponential to power-law intermeeting time in mobile ad hoc networks," IEEE/ACM Transactions on Networking, vol. 17, no.5, pp.1578-1591, 2009.

[13] P. J. Burke, “The output of a queuing system,” Operations Research, vol.4, no.6, pp.699-704, 1956.

[14] Shanghai Taxi Trace Data [Online], Available: http://wirelesslab.situ.edu.cn. 


\section{Figures:}

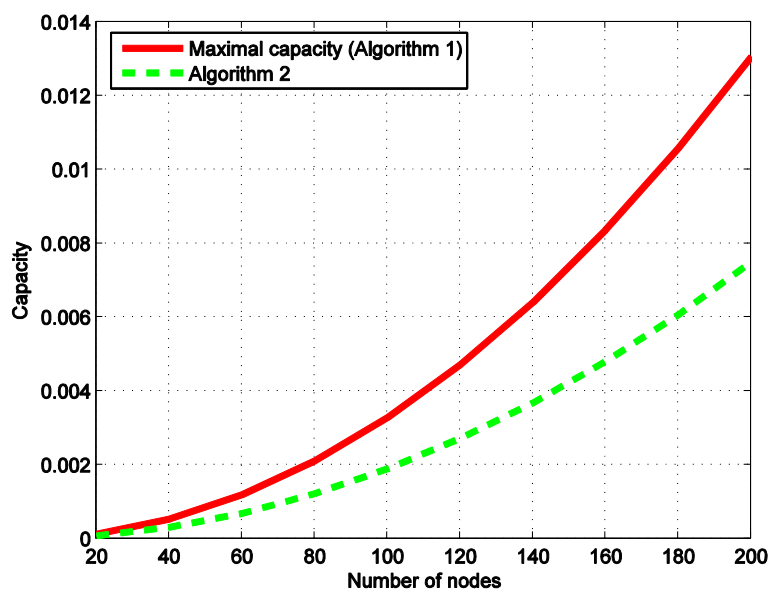

Fig.1 The capacity vs. the number of nodes.

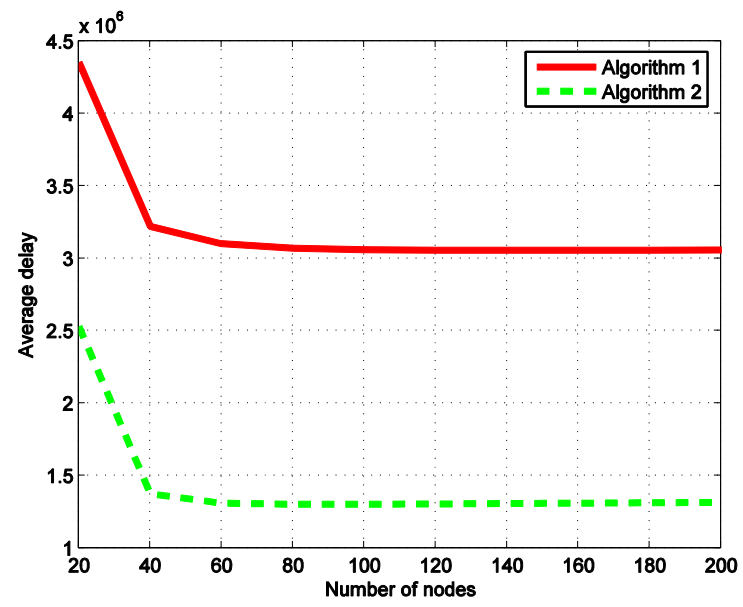

Fig.2 The average delay vs. the number of nodes.

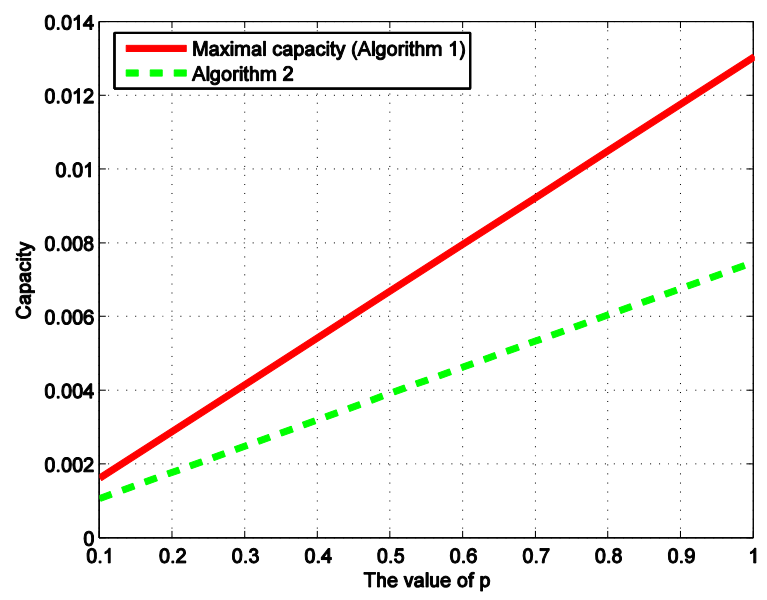

Fig.3 The capacity vs. social selfishness. 


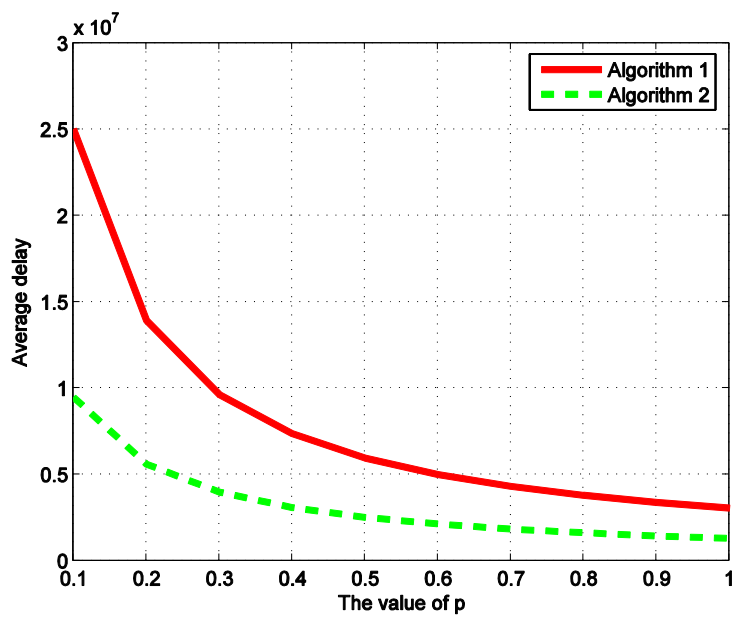

Fig.4 The average delay vs. social selfishness. 